\title{
Acknowledgement to Reviewers of Entropy in 2017
}

\author{
Entropy Editorial Office
}

MDPI AG, St. Alban-Anlage 66, 4052 Basel, Switzerland

Published: 15 January 2018

Peer review is an essential part in the publication process, ensuring that Entropy maintains high quality standards for its published papers. In 2017, a total of 684 papers were published in the journal. Thanks to the cooperation of our reviewers, the median time to first decision was 26.98 days and the median time to publication was 53.5 days. The editors would like to express their sincere gratitude to the following reviewers for their time and dedication in 2017:

$\begin{array}{ll}\text { Abah, Obinna } & \text { Li, Zhixiong } \\ \text { Abbas, Ali } & \text { Lian, Wenzhao } \\ \text { Abbas, Ali E. } & \text { Liang, Chao } \\ \text { Abbot, Maysam } & \text { Liang, Chiu-kuo } \\ \text { Abd-Alhameed, Raed } & \text { Liao, Bolin } \\ \text { AbdAllah, Eslam G. } & \text { Lieb, Elliott } \\ \text { Aboalayon, Khald } & \text { LIEBMAN, Joel F. } \\ \text { Abrams, Cameron } & \text { Liemert, Andre } \\ \text { Abutayeh, Mohammad } & \text { Lietman, Thomas M } \\ \text { Acedo, Luis } & \text { Lima, J.A.S. } \\ \text { Acharya, U Rajendra } & \text { Lima, Markus } \\ \text { Adams, Stefan } & \text { Lin, Chih-Hong } \\ \text { Adelantado, Ferran } & \text { Lin, Hong } \\ \text { Adesso, Gerardo } & \text { Lin, Junlin } \\ \text { Afonso, Clito } & \text { Lin, Pei-Feng } \\ \text { Agop, Marciel } & \text { Lin, Shih-Chun } \\ \text { Aguiar, Marcus } & \text { Lin, Yu-Jau } \\ \text { Aguilar, Cristina } & \text { Linn, Jimmy B. } \\ \text { Aguirre-Salado, Carlos A. } & \text { Lio, Yuhlong } \\ \text { Ahmad, Fayyaz } & \text { Lipniacki, Tomasz } \\ \text { Ahmadi, Mehdi } & \text { Lipowski, Adam } \\ \text { Ahmadi, Pouria } & \text { Liu, Chongxin } \\ \text { Ahmadlouydarab, Majid } & \text { Liu, Chunhua } \\ \text { Ahmed, Mosabber Uddin } & \text { Liu, Fang } \\ \text { Ahn, Choon Ki } & \text { Liu, Jain-Shing } \\ \text { Ajgl, Jiří } & \text { Liva, Gianluigi } \\ \text { Akbar, Muhammad } & \text { Livadiotis, George } \\ \text { Akbarzadeh, Hamid } & \text { Lizier, Joseph } \\ \text { Akih-Kumgeh, Ben } & \text { Ljubenko, Andrej } \\ \text { Aktaruzzaman, Md } & \text { Llopis, Rodrigo } \\ \text { Akyol, Emrah } & \text { Lo Franco, Rosario Lo } \\ \text { Ala-Nissilä, Tapio } & \text { Loaiciga, Hugo A. } \\ \text { Al-Ashwal, Waddah A. } & \text { Logsdon, Sally D. } \\ \text { Alberto, Perea Moreno } & \text { Loiola, Murilo Bellezoni } \\ \text { Alcaine, Alejandro } & \text { Lombardi, Olimpia } \\ \text { Alconero, Patricia Luis } & \text { Lombardo, Maria Carmela } \\ \text { Alexeev, Alexander } & \\ & \end{array}$


Alfonso, Leonardo

Ali, Sean A.

Almgren, Khaled

Alonso, Daniel

Alonso, Joan F

Alonso, Miguel A.

Alonso-Serrano, Ana

Alvarez-Alonso, Antonio

Amado, Miguel

Amaguchi, Hideo

Amari, Shun-ichi

Amigó, José

Amiri, Mehdi

Ammari, Habib

Ammon, Martin

Amza, Catalin

Andaluz, Joaquín

Anderson, Amy D.

Ando, Tadashi

Aneziris, Olga

Angstmann, Christopher

Angulo, Juan C.

Angulo-Brown, F

Annila, Arto

Anselmet, Fabien

Antal, Peter

Antonopoulos, Angelos

Antuchevičienè, Jurgita

Apfelbaum, E. M.

Apiecionek, Łukasz

Appadoo, Srimantoorao S.

Aprea, Ciro

Arce, Pedro F.

Archer, Reginald

Ardenghi, Juan Sebastián

Arecchi, Fortunato Tito

Argyros, Ioannis

Arima, Takashi

Arizmendi, Miguel

Arndt, Markus

Aros, Rodrigo

Arroyo, David

Arsenić, Ilija

Arthanari, Tiru

Artiemjew, Piotr

Aryal, Jagannath

Asad, Jihad

Asadi, Behzad

Asboth, Janos K.

Ashlock, Dan

Ashton, Neil

Assar, Saïd

Assecondi, Sara

Asselmeyer-Maluga, Torsten
Longo, Sandro

Lopes, António

Lopes, Artur O.

López-Presa, José Luis

López-Ruiz, Ricardo

Lorenz, Chris

Lorenz, Ralph D.

Losada, Marcelo

Lostado, Ruben

Lu, Jianfeng

Lu, John C.-C.

Lu, Rongxing

Luca, Adrian

Lucas, Gale

Lucia, Umberto

Luengo, David

Luger, George F.

Lunev, Artem V

Lunghi, Alessandro

Lunin, Oleg

Luo, Chaomin

Luo, Jia-Ning

Luo, Jianwen

Luo, Shunlong

Luongo, Orlando

Lupo, Cosmo

Lux, Thomas

Lv, Songjun

Ly, Alexander

Lyashenko, Eugenia

Lyra, Marcelo

Lyubushin, A. A.

Lyuu, Yuh-dauh

Ma, Andy Jinhua

Ma, Tinghuai

Ma, Xiaoli

Macedo, Pedro

Machado, Tenreiro

Machta, Jonathan

Maciejewski, Andrzej J

Mackiewicz, Michal

MacLellan, Michael

Mady, Carlos E.k.

Magenes, Giovanni

Mahdavi, Mahboobe

Mahian, Omid

Maina, Fadji Zaouna

Maiorino, Angelo

Makse, Hernán A.

Malakhov, Dmitri

Malgaretti, Paolo

Malvoni, Maria

Mammone, Nadia

Mancini, Stefano 
Astaras, Alexandros

Aste, Andreas

Atangana, Abdon

Atoche, Alejandro Castillo

Aubry, Augusto

Aurell, Erik

Ausloos, Marcel

Awad, Ali Ismail

Awan, Zohaib

Awrejcewicz, Jan

Ay, Nihat

Ayele, Yonas Zewdu

Azami, Hamed

Baaj, Hassan

Baaliña Insua, Álvaro

Baas, Andreas

Badescu, Viorel

Badin, Gualtiero

Bagnato, Luca

Baillie, Richard T.

Balasis, Georgios

Baldini, Gianmarco

Balduzzi, David

Baleanu, Dumitru

Balieu, Romain

Bamba, Kazuharu

Ban, Adrian I.

Ban, Masashi

Bandt, Christoph

Banik, Suman K

Bañuls, Mari Carmen

Barato, Andre C.

Baraviera, Alexandre Tavares

Barberis-Blostein, Pablo

Barbosa, Ramiro S.

Bargh, Mortaza Shoae

Bariviera, Aurelio F.

Bariviera, Aurelio Fernández

Barkley, Dwight

Barnett, Lionel

Baroth, Julien

Barr, Richard G.

Barralon, Pierre

Barrett, Adam B.

Baruník, Jozef

Basaran, Cemal

Bashivan, Pouya

Bashkirtseva, Irina

Batalla, Jordi Mongay

Baumert, Mathias

Bayomy, A. M

Bechinger, Clemens

Beckwith, Andrew Walcott
Mandal, Abhijit

Mandic, Danilo

Mangili, Francesca

Manis, Georgios

Maniu, Silviu

Man'ko, Vladimir

Mann, Richard P.

Mann, Robert

Mann, Robert B.

Manning, Gerald S.

Mantzaris, Alexander V.

Manzanares, José Antonio

Marcelli, Thierry

Marcellin, Michael W.

Marcolli, Matilde

Marcon, Eric

Marconi, Umberto Marini Bettolo

Marek-Crnjac, Leila

Maren, Alianna J.

Mari, Andrea

Marijuán, Pedro

Marinazzo, Daniele

Marinescu, Catalina

Marini, Gustavo

Marković, Dimitrije

Marmelat, Vivien

Marmur, Abraham

Marozas, Vaidotas

Marquet, Pablo

Marriott, Paul

Marriott, Paul K.

Marsland, Stephen

Martalo, Marco

Martalò, Marco

Martín, Manuel Jesús Cobo

Martinez, Ramses V.

Martínez-Rodrigo, Arturo

Martinez-Valdes, Eduardo

Martinopoulos, Georgios

Martyushev, L.M.

Martyushev, Leonid

Marujo, Luis

Maruta, Kazuki

Marzbanrad, Faezeh

Maschke, Bernhard

Masoumi, Samira

Mastriani, Mario

Mastroeni, L.

Masuda, Naoki

Mata, Maria Eugénia

Matei, Ani

Mateo-Lázaro, Jesús

Mateos Roco, José Miguel 
Bee, Marco

Beer, Randall

Beggs, John M.

Behar, Joachim

Bekiros, Stelios

Bellos, Evangelos

Belman-Flores, J.M.

Belonosko, Anatoly

Belsare, A. D.

Beltra, Rafael Lahoz

Benato, Alberto

Benatti, Fabio

Benedetto, Francesco

Bengtsson, Ingemar

Benim, Ali Cemal

Ben-Naim, Arieh

Benninger, Daniel

Beranek, Ladislav

Berens, Philipp

Beretta-Piccoli, Matteo

Bermudez, Marcel

Bernardi, Giulio

Berthoumieu, Yannick

Berto, Filippo

Bertschinger, Nils

Besagni, Giorgio

Beşdok, Erkan

Beyerer, Jürgen

Bhattacharia, Sanjoy

Bhotto, Md. Zulfiquar Ali

Biagetti, Giorgio

Bialecka-Florjańczyk, Ewa

Bianchi, Giuseppe

Bianchini, Cosimo

Bianco, Vincenzo

Bianucci, Marco

Bienertová-Vašků, Julie

Biondo, Alessio Emanuele

Bird, Simeon

Bishop, Mark

Bisi, Marzia

Bisio, Alessandro

Biswas, Soumyajyoti

Bithas, Petros S.

Bizarro, João P. S.

Blanche, Pierre-alexandre

Blasco, Jorge

Blázquez-Salcedo, Jose Luis

Blecich, Paolo

Blencowe, Miles

Bloch, Matthieu

Bloem, Peter

Bo, Tan
Matera, Mauricio

Matilla-García, Mariano

Matone, Marco

Matsoukas, Themis

Matsumoto, Ryutaroh

Matsuta, Tetsunao

Matúš, František

Maunder, Rob

Mauri, Roberto

Mauro, John

Maybank, Steve

Mayer, Christopher

Mazzola, Guglielmo

McClure, James

McCulloch, Mike

McDonald, Kirk T.

McGregor, Simon

McGuire, Michael

McKenzie, Erica

Medved, Vladimir

Meintanis, Simos G.

Melis, Roberta

Melkikh, Alexey V.

Meloni, Carlo

Meloni, Simone

Melvin, William L.

Mendez, Martin Oswaldo

Méndez, Vicenç

Menendez, Hector

Menéndez, Héctor D.

Meo, Marianna

Merabia, Samy

Merletti, Roberto

Mesa Sanchez, Oscar Jose

Mestdag, Tom

Metcalfe, Stan

Mezei, József

Michailidis, Panagiotis

Michalski, Radosław

Michel, Florent

Michieletto, Davide

Mihailovic, Dragutin

Milani, Simone

Miłkowski, Marcin

Miller, Luis

Milne, Bruce

Milone, Candida

Milton, Kimball

Mimoso, José Pedro

Minea, Alina Adriana

Ming, Dengming

Miozzo, Marco

Miranda, Jose Garcia Vivas 
Bobbio, Andrea

Bode, Leticia

Bogaert, Jan

Bogdan, Piotr

Bogner, Konrad

Boguniewicz, Joanna

Bondarescu, Ruxandra

Bopp, Fritz

Bordallo, Heloisa N.

Borgonovi, Fausto

Boriga, Radu

Bormashenko, Edward

Borodich, Feodor M.

Borovcnik, Manfred

Bosiljka, Tadic

Boškoski, Pavle

Bossomaier, Terry

Boström, Kim

Bosyk, Gustavo Martin

Bottazzi, Giulio

Boubchir, Larbi

Boucheron, Stéphane

Bougheas, Spiros

Bourgine, Paul

Bourguignon, Marcelo

Bousia, Alexandra

Boyadzhiev, Khristo N.

Boyom, Michel

Boyom, Michel Nguiffo

Bozdogan, Hamparsum

Brandner, Kay

Branicki, Michal

Braun, James

Bravetti, Alessandro

Brelsford, Christa

Bresme, Fernando

Brigham, Katharine

Brini, Emiliano

Broadbridge, Philip

Brodie, Ian M.

Brody, Justin

Bross, Shraga

Brouers, Francois

Browne, Dan

Brunton, Bingni Wen

Bruschi, David Edward

Bub, Jeffrey

Buchmeister, Borut

Bui, Nicola

Bukal, Mario

Bukkapatnam, Satish T.S.

Burini, Diletta

Burtini, Giuseppe

Buza, Krisztian
Miret-Artés, Salvador

Miro, Shorash

Mishra, Ashok

Miśkiewicz, Janusz

Mitsche, Dieter

Mobed, Nader

Mohajeri, Nahid

Mohammad-Djafari, Ali

Mohler, M. Jane

Mohssen, Magdy

Molinaroli, Luca

Molinero, Xavier

Monaco, Vinnie

Mondejar, Maria E.

Mondelli, Marco

Montanari, Gian Carlo

Montesi, Danilo

Montessori, Andrea

Montufar, Guido F.

Moon, Jongsub

Mora, Higinio

Mora, Thierry

Morabito, Francesco Carlo

Morales Luna, Guillermo

Moral-Muñoz, José Antonio

Moreno Arboleda, Francisco Javier

Moretto, Enrico

Morgenthaler, Stephan

Morison, Gordon

Morley, Bruce

Moroni, Monica

Morosuk, Tatiana

Moroz, Adam

Morrone, Biagio

Morrone, Pietropaolo

Moshou, Dimitrios

Mota, Alzira

Mountrouidou, Xenia

Mousa, Amr

Moustakidis, Charalampos

Mück, Wolfgang

Mueller, Markus

Muga, J. Gonzalo

Mukherjee, Partha Sarathi

Mukherjee, Pritam

Mukherjee, Sayan

Mulder, Joris

Müller-Vahl, Kirsten R.

Mungan, Carl E.

Muñoz, José J.

Murtagh, Fionn

Musa, Sarhan M.

Muscato, Orazio

Musgrave, Ian 
Cabboi, Alessandro

Cabral, João M. G.

Cabral, Pedro

Cabrerizo, Francisco Javier

Cacciatori, Sergio L.

Cafaro, Carlo

Cai, Yi-Fu

Caiado, Camila

Cailliez, Fabien

Caire, Giuseppe

Caleffi, Marcello

Callico, Gustavo M.

Calvo Hernandez, A

Camarena-Martinez, David

Campo Vázquez, Celeste

Campo, Adrià Tauste

Cano, Alberto

Canovas Pena, Jose S.

Cao, Bingyang

Cao, Jinde

Capecci, Elisa

Caporale, Guglielmo Maria

Caprara Vivoli, Valentina

Caraiani, Petre

Caramés, Tiago Fernández

Caravelli, Francesco

Carbia Carril, José

Carbone, Anna

Cardoso, Jaime

Carlini, Maurizio

Carminati, Maria Chiara

Carmona, Jose Manuel

Carpena, Pedro

Carpi, Laura

Carsteanu, Alin Andrei

Carter, Alan

Carvalho, André De

Carvalho, Leonor

Carvalho, Paulo

Casalino, Gabriella

Casarin, Roberto

Castellano, Javier García

Castellano, Nicola Giuseppe

Castiglioni, Paolo

Castillo Sequera, José Luis

Catarino, Isabel

Cattani, Carlo

Cavalcante, Charles Casimiro

Cavalcanti, Eric G.

Cejnar, Pavel

Celesti, Antonio

Cembranos, J. A. R.

Cencini, Massimo
Musslimani, Ziad

Myers, Chris

Myong, R.S.

Nabity, James

Nadin, Mihai

Nagarajan, Raghavan

Naik, Ganesh

Nakamura, Tadas K.

Nakao, Hiroya

Nalepa, Jakub

Nanni, Loris

Napoli, Alessandro

Napolitano, Francesco

Naranjo, Lizbeth

Narnhofer, Heide

Narusawa, Uichiro

Nassar, Antonio

Nasser, Rajai

Nasuto, Slawomir

Naudts, Jan

Navara, Mirko

Navarro-Mesa, Juan L.

Nelson, Kenric P.

Nemś, Magdalena

Neri, Izaak

Nestorovich, Ekaterina

Netočný, Karel

Newell, Karl

$\mathrm{Ng}$, Derrick Wing Kwan

$\mathrm{Ng}$, Jason

Nguyen, Nha

Nian, Jun

Nicolaou, Nicoletta

Nicolis, Grégoire

Nielsen, Heber

Nieto, Juan J.

Nigmatullin, Ramil

Nijsure, Yogesh

Nikas, George K.

Nikkhah-Moshaie, Roozbeh

Nikolaev, Alexander

Nikulov, Alexey

Nino-Ruiz, Elias D.

Niro, Alfonso

Nissen, Ivor

Niven, Robert

Nivre, Joakim

Nock, Richard

Noh, Gosan

Noh, Jae Dong

Nojiri, Shin'ichi

Nolfi, Stefano

Nosonovsky, Michael 
Cerbino, Roberto

Cerello, Piergiorgio

Cerulo, Luigi

Chacón-Acosta, Guillermo

Chai, Rifai

Chakraborty, Biman

Chalishajar, Dimplekumar

Chamberlin, Ralph

Chan, Chung

Chan, Felix

Chander, Harish

Chang, Lo-Bin

Chang-Jian, Cai-Wan

Chaplain, Mark

Charalampos, Pitas

Charles-Cadogan, Godfrey

Chathoth, Suresh Mavila

Chau, Kwok-wing

Chechkin, Aleksei

Chen, Badong

Chen, Binqiang

Chen, Bor-Sen

Chen, Boxing

Chen, Chun-Chi

Chen, Der-chin

Chen, Hongshu

Chen, I-Te

Chen, Jianyong

Chen, Lingen

Chen, $\mathrm{Lu}$

Chen, Mu-Song

Chen, Shyi-Ming

Chen, Ting-li

Chen, Wenxi

Chen, $\mathrm{Xi}$

Chen, Yanguang

Chen, Yanling

Chen, Yuhua

Chen, Zonghai

Cheng, Changqing

Cheng, Zhiyong

Chernodub, Maxim

Chesneau, Xavier

Chew, Lockyue

Chi, Sien

Chiachio, Manuel

Chiarelli, Piero

Chiatti, Leonardo

Chibbaro, Sergio

Chicharro, D.

Chiementin, Xavier

Chien, Feng-Tsun

Chih, Mingchang

Chmiel, Anna
Novitsky, Denis V

Nurzaman, Surya

Oblak, Daniel

Obuchi, Tomoyuki

Ocłoń, Pawel

Odgaard, Peter Fogh

Odintsov, Sergei

Odqvist, Joakim

Oechtering, Tobias

Oh, Simon

Oikonomou, V.K.

Oizumi, Masafumi

Ojovan, Michael

Okon, Elias

Olbrich, Eckehard

Oliveira Mimoso, José Pedro

Oliveira, Elismar R.

Oliveira, Santiago Del Rio

Oliveto, Giuseppe

Olmos, Pablo M.

Olsen, Lars Ole Ronnow

Omachi, Shinichiro

Oneto, Luca

Onken, Arno

Oprea, Iuliana

Orcioni, Simone

Orciuoli, Francesco

Ordonez, Gonzalo

Oreshkov, Ognyan

Ortega, Guillermo J

Ortiz De Zárate, José M.

Ortiz-García, Mario

O'Shaughnessy, Douglas

Ossadtchi, Alexey

Ostoja-Starzewski, Martin

Oświęcimka, Paweł

Oszust, Mariusz

Ou, Congjie

Ouerdane, Henni

Ouldridge, Thomas

Ovchinnikov, Igor

Ozel, Omur

Paez-Hernandez, Ricardo

Pahle, Jürgen

Paja, Wieslaw

Palma, G.Massimo

Palyulin, Vladimir Vladimirovich

Panagiotidis, Theodore

Panagiotopoulos, Alexandros

Pandey, M.D.

Pang, Xiaodan

Pankratov, Andrey

Pantaleo, Antonio

Pantaleo, Antonio Marco 
Cho, Hwan-Gue

Cho, Joon Ho

Chodera, John D.

Choi, Jun-Won

Choo, Raymond

Chotorlishvili, Levan

Christen, Thomas

Christodoulides, Paul

$\mathrm{Chu}$, Yongbin

Chuang, Michael

Chui, Kwok Tai

Chun, Kwok Pan

Ciaccio, Edward J.

Ciaramella, Angelo

Ciuonzo, Domenico

Claeskens, Gerda

Clemente, Riccardo

Cleuren, Bart

Clisby, Nathan

Cluni, Federico

Cockshott, William

Codetta-Raiteri, Daniele

Coecke, Bob

Cohen, Eliahu

Colangelo, Gianpiero

Coles, Patrick

Colonius, Fritz

Comes, Calin

Comez, Lucia

Como, Giacomo

Congedo, Paolo Maria

Connor, Richard

Consoulas, Christos

Contreras-Reyes, Javier E.

Cooke, Thomas J.

Copot, Cosmin

Corani, Giorgio

Coro, Gianpaolo

Corominas-Murtra, Bernat

Cortés López, Juan Carlos

Cosma, Georgina

Costa, Cesar Da

Costa, Mário

Costa-jussà, Marta R.

Covelli, Carmine

Craus, Mitică

Crepeau, John

Crippa, Paolo

Cristelli, Matthieu

Croca, José Nunes Ramalho

Croke, Sarah

Crokidakis, Nuno

Crosignani, Bruno
Pantazis, Yannis

Panzeri, Stefano

Pap, Gyula

Papathanasiou, Jason

Pappalardo, Luca

Pappu, Chandra

Paradisi, Paolo

Park, Choon-Su

Park, Hyunggyu

Park, Nohhyun

Parra, Ruben D.

Parvan, Alexandru S.

Passarella, Francesca

Passon, Oliver

Pastore, Raffaele

Pastorello, Davide

Paternostro, Mauro

Patrascu, Andrei

Paunkovic, Nikola

Pavelka, Michal

Pavlos, George

Pavsic, Matej

Paya, Luis

Payaró, Miquel

Pazos, Alejandro

Pearle, Philip

Pecht, Michael Gerard

Pei, Zongrui

Peinke, Joachim

Pejaś, Jerzy

Peláez-Moreno, Carmen

Pelorosso, Raffaeile

Pendrill, Leslie

Peng, Linyu

Peng, Weiwen

Pennini, F.

Pennisi, Sebastiano

Perarnau-Llobet, Martí

Perc, Matjaz

Perdigão, Rui A. P.

Pereira, Mário

Peretti, Christian De

Pérez, Agustın-Agüera

Pérez-Madrid, A.

Peris, Bernardo

Perivolaropoulos, Leandros

Perkins, Theodore

Perlick, Volker

Perram, John William

Perron, Aurelien

Peshkov, Ilya

Pesta, Michal

Petrellis, Nikos 
Cruz-Hernández, César

Cudennec, Christophe

Cuesta-Frau, David

Cui, Xingran

Cummins, Fred

Curado, Evaldo M. F.

Cutler, Adele

Czinner, Viktor G.

Da Luz, Marcos Gomes Eleutério

Da Silva, Mario Marques

D'Abramo, Germano

Dagdug, Leonardo

Dai, Weihui

Daivis, Peter J.

D'Andreagiovanni, Fabio

Danner, Simon M

Dantcheva, Antitza

Dappiaggi, Claudio

Dariusz, Wrzesiński

Darvishi Kamachali, Reza

Das, Manohar

Dassios, Ioannis

Dassios, Ioannis K.

Datcheva, Maria

Datta, Jyotishka

Daum, Fred

David, Sergio

Davila-Velderrain, Jose

Davis, Sergio

Daw, Stuart

Dawid, Philip

De Andrade Alves, Luiz Gustavo

De Araujo, Jose Carlos

De Arcangelis, Lucilla

De Bragança Pereira, Carlos Alberto

De Brevern, Alexandre

De Capitani, Lucio

De Castro, Mario

De Chiara, Gabriele

De Giuli, Maria Elena

De Jong, Wil

De Kerret, Paul

De Leon, Manuel

De Meo, Pasquale

De Miras, Juan Ruiz

De Oliveira Júnior, Silvio

De Pascale, Andrea

De Pascali, Chiara

De Rosa, Sergio

Debeir, Olivier

Debowski, Lukasz

Dębski, Adam

DeDeo, Simon

Degaetano-Ortlieb, Stefania
Pétritis, Dimitri

Phinyomark, Angkoon

Piantadosi, Steven T.

Piasecki, Krzysztof

Pichl, Lukas

Pietronero, Luciano

Pimenta Dinis, Maria Alzira

Pinho, Armando J.

Pinoli, Jean-Charles

Pinto, Carla

Piovani, Duccio

Pires, Cesaltina

Pirner, Hans Jürgen

Piskorski, Jarosław

Pistoia, Francesca

Pivoluska, Matej

Plakandaras, Vasilios

Planat, Michel

Plank, Michael J.

Plastina, Francesco

Plastino, Angel

Plastino, Angelo

Platt, Trevor

Pławiak, Paweł

Pleimling, Michel

Plesch, Martin

Poelzing, Steven

Poet, Ron

Pogrebnjak, Alexander

Pohl, Christine

Pokrajac, David

Poletti, Dario

Polettini, Matteo

Polimeno, Antonino

Politi, Antonio

Pompe, Bernard

Poncet, Sébastien

Pooranian, Zahra

Pop, Ioan

Popescu, Mihail N.

Porporato, Amilcare

Porta, Alberto

Post, Thierry

Poti, Valerio

Potirakis, Stelios

Poveda, German

Povstenko, Yuriy

Pradas, Marc

Prasath, V. B. Surya

Prażmowska, Małgorzata

Prażmowski, Krzysztof

Prehl, Janett

Pressé, Steve

Preziosi, Valentina 
Dehmer, Matthias

Deiters, Ulrich

Del Campo, Adolfo

Delgado, Manuel

Della Rossa, Fabio

Delogu, Francesco

Del-Val-Noguera, Elena

DeMazumder, Deeptankar

Demetrius, Lloyd

Deng, Jeremiah

Deng, Mingcong

Dennard, Linda

Desbois, Dominique

Desmet, Bernard

Dette, Holger

Deufemia, Vincenzo

Dewan, Ashraf

Di Gravio, Giulio

Di Crescenzo, Antonio

Di Francescomarino, Chiara

Di Martino, Ferdinando

Di Nola, Antonio

Di Vita, Andrea

Di Zio, Simone

Dieks, Dennis

Dienes, Zoltan

Diep, Hung T.

Dijkstra, Bauke W.

Dima, Mihai-Octavian

Dimitriou, Dimitrios

Dimitrov, Alexander G.

Dinis, Luis

Dinneen, Michael J

Dionisio, Andreia

Divigalipitiya, Prasanna

Dixit, Purushottam D.

Dobrovolny, Hana M.

Dodd, Ian C.

Dodig-Crnkovic, Gordana

Dolan, Brian

Dolfin, Marina

Domingues, Pedro

Dong, Boxiang

Dong, Daoyi

Dong, Yiqiu

Donner, Reik

Doran, Derek

Dove, Guy

Dreibholz, Thomas

Dressel, Justin

Dreżewski, Rafał

Duan, Jinming
Priesemann, Viola

Proesmans, Karel

Protzner, Andrea B.

Pruessner, Gunnar

Psannis, Konstantinos

Puerta Callejón, José Miguel

Pulido, Inmaculada

Qiao, Yuansong

Qin, Sanbo

Qualls, Whitney A.

Quarati, Piero

Quax, Rick

Queiros, Silvio M. Duarte

Quintero-Quiroz, Carlos

Quitadamo, Lucia

Raboso, Mariano

Radac, Mircea-Bogdan

Radak, Brian

Raddant, Matthias

Radenković, Slavko

Radica, Gojmir

Radulovic, Jovana

Rafał, Marcin Laskowski

Ragulskis, Minvydas

Rahnejat, Homer

Rajendra Acharya, U.

Rajsekhar, Deepthi

Rakhshan, Ali

Ramachandran, K. I.

Ramampiaro, Heri

Ramirez, Israel Reyes

Ramírez-Reyes, Abdiel

Ramos, Célia M. Q.

Ramos, Rubens Viana

Ramsey, Vincent K.

Ran, Shiju

Rapaport, William

Rashidi, Mohmad Mehdi

Rashkovits, Rami

Rathgeber, Andreas

Rathie, Pushpa

Raudino, Antonio

Rauh, Johannes

Ravelli, Flavia

Ravelli, Silvia

Ravi, Ananth

Raz, Oren

Reeke, George N.

Reichardt, Christian

Reichhardt, Charles

Reis, Pedro Miguel Ramos

Ren, Jinchang 
Duann, Jeng-Ren (J.R.)

Duarte-Mermoud, Manuel A.

Dubenko, Igor

Dudczyk, Janusz

Dukka, KC

Dumont, Remi

Dungey, Mardi

Duong, Quang Trung

Dupuis, Frédéric

Dutton, Zachary

Dwivedi, Dipankar

Dworak, Paweł

Dziarmaga, Jacek

Ebaid, Abdelhalim

Eddy, Brian

Ediriweera, Sisira

Egolf, Peter W.

Eibenberger, Sandra

Eisert, Jens

Eleuch, Hichem

Elfadaly, Fadlalla

Ellahi (R. Ellahi), Rahmat

Ellerma, David

ElMoslimany, Ahmad

Elsalloukh, Hassan

El-Shahat, Adel

Elsinger, Helmut

Eltayeb, Mohammed E

Elwakil, Ahmed S.

Elze, Hans-Thomas

Emerson, Joseph

Engelbrecht, Kurt

Enßlin, Torsten

Entin-Wohlmann, Ora

Escassut, Alain

Escudero, Javier

Esfahanian, Mahdi

Espinosa, Maria

Esposito, Massimiliano

Esquivel, Rodolfo O.

Essafi, Karim

Estellé, Patrice

Estrada, Ernesto

Evans, Martin

Evans, Michael

Expert, Paul

Faber, Manfried

Faes, Luca

Faggini, Marisa

Falcucci, Giacomo

Falniowski, Fryderyk

Fan, Pingyi

Fan, Wenke

Fang, Yinfeng
Renevey, Philippe

Rensburg, E.J. Janse Van

Requardt, Manfred

Resinas, Manuel

Rétvári, Gábor

Revell, Alistair

Reyes, José Santos

Reyzin, Lev

Rheem, Jaeyeol

Rhudy, Matthew

Ribeiro, Haroldo

Ricciuti, Costantino

Richman, Joshua

Richmond, Christ D.

Richter, Hendrik

Richter, Wolf-dieter

Ridgway, Sam

Ridolfi, Elena

Riechers, Paul M.

Riechert, Uwe

Riihonen, Taneli

Rinaldo, Roberto

Ringbauer, Martin

Rio, Carlos

Rios, Miguel

Riquelme, Adrián

Risse, Marian

Ritort, Fèlix

Rivero, Cristian Rodriguez

Rodger, James A.

Rodrigues, Alexandre A. P.

Rodrigues, João

Rodrigues, Maria Manuela Fernandes

Rodriguez, Jose M.

Rodríguez, Juan J.

Rodriguez, Leo

Rodríguez, Rosa M.

Roe, Daniel R.

Roffo, Giorgio

Roger, Sandra

Rogers, David

Rogosin, Sergei

Roldan, Edgar

Roldán, Édgar

Romagnoli, Pierre Paul

Romano, Elvira

Romera, Elvira

Romero-Troncoso, Rene De Jesus

Rominger, Andrew J.

Rooh Khurram, Rooh

Rosas, Fernando

Rosić, Bojana

Rossi, Luca

Rosu, Haret 
Farrell, Max

Fasano, Matteo

Federolf, Peter

Fedorov, Aleksey

Fei, Cheng-wei

Feidt, Michel

Felice, Domenico

Felisberto, Paulo

Fellouris, Georgios

Feng, Wanpeng

Feng, Yu

Feng, Yunlong

Fereidountabar, Amirhossein

Ferracuti, Francesco

Ferrando, Riccardo

Ferrara, Massimiliano

Ferraro, Alessandro

Ferreira, Eduarda Pinto

Ferrero, Alessandro

Ferro, Marcello

Fiedor, Paweł

Figliola, Alejandra

Figlus, Tomasz

Figueiras, Edite

Filatrella, Giovanni

Fillion-Gourdeau, François

Fink, Gabriel

Finn, Conor

Fiorentino, Mauro

Fiori, Simone

Fischer, Franz Dieter

Fischer, Matthias J.

Fistola, Romano

Florea, Olivia Ana

Fong, Silas L.

Forbes, Angus G.

Forestier, Germain

Fortuna, Luigi

Fosgerau, Mogens

Fotouhi, Abbas

Fouquier D'Hérouël, Aymeric

Fradin, Cécile

Francisco, Caamaño Isorna

Frank, S

Fränti, Pasi

Franzosi, Roberto

Freddi, Alessandro

Freudenberger, Jürgen

Friedrich, Tobias

Friston, Karl

Froese, Anna

Fronsdal, Christian

Fu, Zuntao

Fujisawa, Hironori
Roterman, Irena

Roth, Bradley J.

Rothkrantz, Leon

Rotter, Ingrid

Rotundo, Giulia

Roumy, Aline

Roussey, Catherine

Rovira, Antonio

Roy, Cerqueti

Roy, Raphaëlle N.

Rozakis, Stelios

Rubi, Miguel

Rubi, Miguel J.

Rubio, F. Javier

Rucco, Matteo

Ruddell, Benjamin

Ruess, Jakob

Ruiz, L. M. Sanches

Ruiz, Magda L.

Russ, John

Ruziboev, Marks

Rwebangira, Mugizi Robert

Sabar, Nasser

Sabín, Carlos

Sáchez Soto, Luis Lorenzo

Sadakane, Kunihiko

Saez-Gomez, Diego

Safaai, Houman

Saffari Pour, Mohsen

Sagawa, Takahiro

Saha, Priya

Said, Salem

Saitoh, Takeshi

Sajeed, Shihan

Salinas, Hugo S.

Samadani, Ehsan

Samani, Afshin

Samek, Wojciech

San Miguel, Adriana

Sanchez, David

Sanchez-Salas, Norma

Sanchez-Taltavull, Daniel

Sandev, Trifce

Sandoval Orozco, Ana Lucila

Sandoval, Leonidas

Sands, David

Sanei, Saeid

Santos, José Joaquim

Santos, Laurita Dos

Santra, Siddhartha

Sanz, Angel S.

Sapora, Alberto

Saravanakumar, Ramasamy

Sarbu, Ioan 
Fujiwara, Yoshi

Fulginei, Francesco Riganti

Fullér, Robert

Fung, Thomas

Furtula, Boris

Gabora, Liane

Gadhoumi, Kais

Gadomski, Adam

Gadouleau, Maximilien

Gagie, Travis

Gajowniczek, Krzysztof

Galam, Serge

Galanis, George

Galeazzi, Roberta

Galperin, Michael

Gama Goicochea, Armando

Gamo, Javier

Gamsjäger, Ernst

Ganguly, Pritam

Gao, Bin

Gao, Song

Gao, Zhongke

García Díaz, Pilar

Garcia, Nuno M.

Garcia-Leon, Manuel

Gardini, Laura

Gargiulo, Gaetano

Garland, Joshua

Gaunt, Robert

Gavrilov, Momčilo

GAVRILUT, Alina Cristiana

Gawron, Piotr

Gay-Balmaz, Francois

Gazeau, Jean Pierre

$\mathrm{Ge}$, Hao

Geiger, Bernhard C.

Gelbstein, Yaniv

Gelbwaser, David

Gencaga, Deniz

Gençay, Ramazan

Gendron, Paul

Genes, Claudiu

Geng, Ruili

Gentili, Claudio

Georgeon, Olivier

Geronimo, Andrew M.

Gervino, G.

Geurdes, Han

Gharabaghi, Bahram

Ghazi-Zahedi, Keyan

Gholamalizadeh, Ehsan

Ghoraani, Behnaz

Ghosh, Abhik

Ghosh, Arnab
Sardina, Gaetano

Sarkisyan-Grinbaum, Edward

Sarlis, Prof. N

Sarracino, Alessandro

Sarri, Gianluca

Sarwate, Anand D.

Sasa, Shin-ichi

Sauer, Tilman

Scalo, Carlo

Scargle, Jeffrey

Schaeffer, Satu Elisa

Scharfenaker, Ellis

Scheibelhofer, Peter

Schilbrack, Kevin

Schilling, Oleg

Schmandt, Bastian

Schmidt, Christian Andrés

Schmidt, Sabine

Schneider, Anton

Schneider, Raimund

Scholkmann, Felix

Scholz, Johannes

Schroeck, Franklin

Schumann, Andrew

Schwarz, Gottfried

Scullion, Tom

Scurrell, Mike

Seghouane, Karim

Selby, John

Semkov, Krum

Senthilnath, J.

Seoane, José A.

Sergeyev, Yaroslav D.

Sergi, Alessandro

Sergio, Curilef

Seshadreesan, Kaushik

Sessa, Salvatore

Severns, Paul M

Shabgard, Hamidreza

Shagrir, Oron

Shahzad, Jawad

Shanmugam, Ram

Shao, Lijing

Shapiro, Jeffrey $\mathrm{H}$.

Sharaev, Maksim

Sharpee, Tatyana

Shawny, Xiao Xiao

She, Xiaohui

Sheikholeslami, Seyed Mahmoud

Sheng, Wenyi

Sherbakov, Sergei

Sheremet, Mikhail

Sheremet, Mikhail Aleksandrovich

Shi, Yongtang 
Giannakis, Konstantinos

Giannerini, Simone

Giannoccaro, Ilaria

Gibson, Edward (Ted)

Gielen, Steffen

Gil, David

Gilbert, Robert

Gill, Richard

Gilli, Manfred

Gillich, Gilbert-Rainer

Giménez, Raimundo Real

Gingrich, Todd

Ginsca, Alexandru Lucian

Gioia, Andrea

Giorgi, Gian Luca

Giuliani, Alessandro

Glavatskiy, Kirill

Glimm, Tilmann

Glowacz, Adam

Gnatyuk, Sergiy

Godang, Romulus

Godinez Tello, Richard

Gogioso, Stefano

Gokaraju, Balakrishna

Golan, Amos

Goldenbaum, Mario

Goldstein, Sheldon

Golubev, Dmitry

Gomez, Ignacio Sebastián

Gomez-Barrero, Marta

Gómez-Déniz, Emilio

Gomez-Pilar, Javier

Gompper, Gerhard

Gonçalves, Hernâni

Gonçalves, Paulo Jorge Sequeira

Gong, Mei

Gontis, Vygintas

Gonzalez, Fernando

Gonzalez-Ayala, Julian

González-de-la-Rosa, Juan-José

González-Díaz, Humberto

González-Rubio, Jesús

Gonzalo-Martín, Consuelo

Goodarzi, Farhad A.

Goodheart, Benjamin

Goodwell, Allison

Gordon, Jeffrey

Górecki, Tomasz

Górski, Marcin

Gorte, Ben
Shikano, Yutaka

Shinohara, Russell Takeshi

Shiskin, Sergei

Shum, Kenneth

Sidorov, Denis N.

Siebenhühner, Felix

Sienkiewicz-Małyjurek, Katarzyna

Siettos, Constantinos

Signorini, Maria Gabriella

Silva Junior, Raimundo

Silva, Cristiana J.

Silva, Luiz Eduardo Virgilio

Silva, Maria Eduarda

Silva, Thiago Henrique

Simani, Silvio

Simon, Joan

Simon, Sergi

Simonetti, Biagio

Simpson, Gideon

Siuly, Siuly

Sivasundaram, Seenith

Siwy, Zuzanna

Skardal, Per Sebastian

Skeppstedt, Maria

Skilling, John

Skotiniotis, Michalis

Skupin, Piotr

Slagmolen, Bram J.J.

Slijepcevic, Sinisa

Slomczynski, Wojciech

Small, Michael

Smart, Paul

Śmieja, Marek

Smirne, Andrea

Smith, Edward R.

Smith, Stefan

Smolić, Ivica

Sohl, Terry

Sole, Patrick

Solis, Francisco

Sommer, Stefan

Son, Seung-Woo

Song, Houbing

Sookhak, Mehdi

Sorguven, Esra

Sothmann, Björn

Soundarajan, Sucheta

Sousa Fragoso, Rui

Souto, André

Spagnolo, Nicolò 
Gosak, Marko

Goto, Shin-Itiro

Gough, John

Goupil, Christophe

Goussis, Dimitris

Goyal, Mania

Goyeneche, Dardo

Gradojevic, Nikola

Gralla, Samuel E.

Gramacy, Robert B.

Gramaglia, Marco

Grazian, Clara

Green, Jason

Green, Michael

Grilli, Jacopo

Grimaldi, Domenico

Grimaldi, Salvatore

Grinbaum, Alexei

Grøn, Øyvind

Gross, Thilo

Groza, Bogdan

$\mathrm{Gu}$, Yina

Guariglia, Emanuel

Guarnieri, Giacomo

Gudowska-Nowak, Ewa

Guendelman, Eduardo

Guenza, Marina

Guirao, Juan Luis García

Gujarati, Purushottam

Gullo, Paride

Guney, Durdu

Gunkel, David J

Guntuboyina, Aditya

Guo, Yuzhu

Gurjeet, Dhesi

Gutiérrez Tobal, Gonzalo César

Gutiérrez-Tobal, Gonzalo C.

Guttmann, Anthony (Tony)

Guyeux, Christophe

Guzowska, Malgorzata Klaudia

Haack, Géraldine

Haba, Zbigniew

Hagerstrom, Aaron

Hale, Jack

Hall, Michael J W

Hamhalter, Jan

Hampel, David

Hamza, Ben

Han, Sunghyu

Hanasaki, Itsuo

Hangos, Katalin

Hannemann-Tamas, Ralf

Hannig, Jan
Sparacino, Giovanni

Sparavigna, Amelia Carolina

Spekkink, Wouter

Spiliotis, Mike

Spina, Damiano

Spohn, Herbert

Spüler, Martin

Squartini, Tiziano

Srikanth, R.

Srinivasa, Arun

Stacey, Blake

Stamatatos, Efstathios

Stamova, Ivanka

Stanciu, Camelia

Starosolski, Roman

Stavrou, Photios A.

Štefaňák, Martin

Stefanatos, Dionisis

Stemler, Thomas

Stephan, Klaas E.

Stewart, Terrence C.

Still, Susanne

Stojkovic, Dejan

Stoltz, Gabriel

Stramaglia, Sebastiano

Strapasson, João E.

Stratton, Peter

Strozzi, Fernanda

Strumiłło, Paweł

Strzalka, Dominik

Stutzer, Michael

Style, Robert

$\mathrm{Su}$, Jianxi

Suarez, Alvaro

Suarez, Elisabet

Subasi, Abdulhamit

Subiantoro, Alison

Sudarsky, Daniel

Suhov, Yuri

Sukharev, Sergei

Sułowicz, Maciej

Sumelka, Wojciech

Sun, Alexander

Sun, Huafei

Sun, Jia Rui

Sun, Jiangwen

Sun, Ke

Sun, Li

Sun, Shiliang

Surana, K.S.

Suyari, Hiroki

Suzuki, Hiroyuki

Suzuki, Reiji 
Haq, Rizwan Ul

Haramaty, Elad

Harandi, Mehrtash T.

Hardal, Ali Ümit Cemal

Harish, Garg

Harko, Tiberiu

Harmandaris, Vagelis

Harre, Michael

Harremoës, Peter

Harrison, Willie

Harshman, Nathan

Harte, John

Hartich, David

Hartmann, Carsten

Haruna, Taichi

Hassan, Mohamed Ahmed

Hassani, Hamed

HATANO, Naomichi

Hatziadoniu, Constantine

Haug-Warberg, Tore

Hauke, Philipp

Haw, Jing-Yan

Hawton, Margaret

Hayajneh, Khaled F.

Hayakawa, Hisao

Hayakawa, Masashi

Hayashida, Morihiro

Heijungs, Reinout

Hellings, Christoph

Hemakom, Apit

Hen, Itay

Henine, Hocine

Hennessy, David

Herdrich, Georg

Hernández-González, Jerónimo

Hernández-Lobato, José Miguel

Herrmann, J. Michael

Herrmann, Klaus

Herwig, Heinz

Hess, Paul

Hettiarachchi, Imali Thanuja

Heydecker, Benjamin

Hiblot, Julien

Hickinbotham, Simon

Hidalgo, Arturo

Hidetoshi, Konno

Hilbert, Martin

Hilbert, Stefan

Hirata, Yoshito

Hiziroglu, Huseyin

Hjorth, Peder

Hofmann, Ralf

Hölbl, Marko

Holik, Federico Hernán
Svoboda, J.

Svozil, Karl

Swendsen, Robert

Syczewska, Malgorzata

Syed, Junaid Nawaz

Szabadics, János

Szabo, Gyorgy

Szabo, Lorand

Szavits-Nossan, Juraj

Szczepanski, Janusz

Sze, Raymond Nung-Sing

Szederkényi, Gábor

Székely, Gábor

Szigeti, Stuart

Szkaliczki, Tibor

Tadmor, Rafael

Takahashi, Daniel

Takahashi, Tatsuji

Takayasu, Hideki

Tal, Ido

Talakoub, Omid

Tamura, Ryo

Tanahashi, Norihiro

Tang, Hong

Tang, Leilei

Tang, Yin

Taniguchi, Shigeru

Tanimoto, Jun

Tar, Jozsef

Tărniceriu, Daniela

Tartaglia, Angelo

Tatarinova, Tatiana V.

Taufer, Emanuele

Tchatoka, Firmin Doko

Telesca, Luciano

Temko, Andriy

Tepljakov, Aleksei

Tervonen, Jouni K.

Theil, Florian

Theis, Dirk Oliver

Thiruvengadam, Magesh

Tilloy, Antoine

Timme, Nicholas

Tkachenko, Denis

Tlelo-Cuautle, Esteban

Toda, Alexis Akira

Todd, Mike

Toffano, Zeno

Toffoli, Tommaso

Tohme, Fernando

Tokuhiro, Akira

Tomar, Vikrant Singh

Tononi, Giulio

Torabi, Mohsen 
Hong, Song-Nam

Hong, Wei-Chiang

Hornung, Grégoire

Horsch, Martin

Horton, W. Travis

Horvát, Emőke-Ágnes

Horvatić, Davor

Hossenfelder, Sabine

Hou, Shuhn-Shyurng

Hovorka, Ondrej

Hron, Karel

Hsiao, Hsu-Chun

Hsiao, Kai-Long

Hsiao, Shen-Fu

Hsu, Li-Chi

$\mathrm{Hu}$, Bei Lok

$\mathrm{Hu}$, Ya-Peng

Huang, Gordon

Huang, Hen-Hsen

Huang, Junyi

Huang, Kejun

Huang, Shih-Feng

Huang, Shoudong

Huang, Wen

Hubbart, Jason

Huber, Marco

Huber, Marcus

Hughes, Keith H.

Humeau-Heurtier, Anne

Huminic, Angel

Huminic, Gabriela

Hung, Hung

Hung, Ling-Yan

Hwang, Chi-Hung

Hwang, Gisuk

Ibl, Martin

Ickowicz, Adrien

Igasaki, Tomohiko

Iglesias, José Roberto

Ikki, Salama

Iliyasu, Abdullah M.

Ince, Robin

Ingber, Lester

Insausti, Xabier

Inuso, Giuseppina

Ipšić, Ivo

Isidro, Jose M.

Islam, Syed

Ito, Sosuke

Izquierdo, Eduardo J.

Izquierdo, Segismundo S.

Izumida, Yuki

Jack, Rachael E.

Jack, Robert

Jacob, Seibu Mary
Toral, Antonio

Torrens, Paul

Torrieri, Giorgio

Toussaint, Udo Von

Tramontana, Fabio

Trancossi, Michele

Trapin, Luca

Travieso-Gonzalez, Carlos M.

Travlos, Ilias

Trevathan, Jarrod

Trevizoli, Paulo Vinicius

Triantaphyllou, Evangelos

Trifonov, Peter V.

Trivellato, Barbara

Trovati, Marcello

Tsai, Chih-Fong

Tsai, Chun-Wei

Tsai, Yao-Hong

Tscharner, Vincent Von

Tse, Ka Kui

Tsinos, Christos G.

Tsonis, Anastasio

Tsyganova, Julia V.

Tuck, Adrian

Tuckerman, Mark

Tura, Jordi

Turkanović, Muhamed

Turki, Sadok

Turner, Brandon

Turner, Mark

Turocy, Theodore

Tušek, Jaka

Uddin, Gazi Salah

Ueshima, Nobufumi

Uffink, Jos

Ullah, Shatif

Unakafov, Anton M.

Unakafova, Valentina

Uohashi, Keiko

Urniezius, Renaldas

Uruba, Václav

Ushizima, Daniela

Vaidogas, Egidijus Rytas

Vaitkus, Andrius

Valdinoci, Enrico

Valencia, José F.

Valenti, Davide

Valenti, Vitor Engracia

Valentino, Eleonora Di

Vallino, Joseph

Valverde-Albacete, Francisco J

Valyrakis, Manousos

Vamvoudakis, Kyriakos

Van Huffel, Sabine

Van Maldeghem, Hendrik 
Jacobi, Ian

Jacobs, Donald

Jacquir, Sabir

Jaeger, Gregg

Jain, Akanksha

Jakus, Damir

James, Ryan

Jang, Gun Ho

Jasra, Ajay

Jauregui, Max

Javarone, Marco Alberto

Jeleń, Lukasz

Jenčová, Anna

Jendrzejczyk-Handzlik, Dominika

Jenkins, Kenneth

Ji, Mingyue

Ji, Sungchul

Jiang, Hao

Jiang, Jingfeng

Jiang, Lanlan

Jiang, Yizhang

Jin, Zhigang

Jog, Varun

Johnson, Sarah J

Jolley, Caroline J.

Jones, Chris

Jones, J Cliff

Jong, Gwo-jia

Jonsdottir, Johanna

Jorgensen, Palle

Jorgensen, Palle E.T.

Joseph, Jojo

Joshi, Siddarth

Joutsijoki, Henry

Jovanovic, Franck

Juárez, Isaac Leobardo Sánchez

Juba, Brendan

Jung, Young-Dae

Jurado, Juan Garrido

Jurkiewicz, Jerzy

Jurman, Giuseppe

Kahirdeh, Ali

Kakar, Muhammad Rafiq

Kalinay, Pavol

Kalogeropoulos, Nikos

Kamidis, Nikolaos

Kamiński, Marcin

Kang, Dae-Ki

Kang, Kyongok

Kang, Wei

Kaniadakis, Giorgio

Kantarcioglu, Murat

Kantardzic, Mehmed M.

Karagrigoriou, Alex
Van Putten, Michel J.A.M.

Van Someren, Maarten

Van Vliet, Marijn

Van Wijland, Fred

Vanapalli, Srinivas

Vandaele, Nico J.

Vargas-Rosales, César

Varshney, Kush R.

Vassiliev, Dmitri

Vazquez-Vilar, Gonzalo

Vedula, Prakash

Velazquez, Luisberis

Vélez Upegui, Jorge Julián

Venegas, Maria

Verda, Vittorio

Verdoolaege, Geert

Vergara, J. David

Vernieri, Daniele

Versteegh, Marijn A. M.

Vesin, Jean-Marc

Vianna, Reinaldo Oliveira

Vicente, Raul

Vickers, Steven

Victor, Jonathan

Vignudelli, Stefano

Vila, Xosé A.

Vilar, Jose

Villani, Marco

Villarroel, Grover Zurita

Villecco, Francesco

Vilmart, Gilles

Virosztek, Dániel

Visentin, Denis

Vitabile, Salvatore

Vitanov, Nikolay

Vitanov, Nikolay V.

Vitányi, Paul

Voit, Eberhard

Volkov, Alexey N.

Volosniev, A. G.

Von Spakovsky, Michael

Von Toussaint, Udo

Vontobel, Pascal O

Vorovencii, Iosif

Vorwerk, Johannes

Vucelja, Marija

Vucetich, Héctor

Vullings, Rik

Vulpe, Alexandru

Vulpiani, Angelo

Wacławczyk, Marta

Wada, Tatsuaki

Wagoner, Jason

Walk, Nathan 
Karakatsanis, L.P.

Karami, Azam

Karasuyama, Masayuki

Karimi, Hamid

Karimi, Nader

Karlgaard, Christopher D.

Karlin, Ilya

Karperien, Audrey L.

Karpov, Evgueni

Karsai, Istvan

Karyotis, Vasileios

Kastner, Ruth

Kateris, Dimitrios

Katkov, Mikhail

Katsura, Hosho

Katunin, Andrzej

Kauranne, Tuomo

Kavic, Michael

Kawalec, Adam

Kawazura, Yohei

Kay, Jim

Kazakis, Nerantzis

Ke, Jia-Hong

Kelbert, Mark

Keller, Karsten

Kelly, James F.

Kenett, Dror Y.

Keogh, Eamonn

Keshavarzian, Sajjad

Khalil, Mohammad

Khalilia, Mohammed

Khan, Ilyas

Khandoker, Ahsan Habib

Kharrazi, Ali

Khlopov, Maxim Yu.

Khrennikov, Andrei

Khurshudyan, Martiros

Killick, Rebecca

Kim, BoHung

KIM, Cheonshik

Kim, Dong Han

Kim, Eun-jin

Kim, Hyunju

Kim, Jennifer A.

Kim, Jung Tae

Kim, Sangdan

Kim, Sang-Hyo

Kimiaghalam, Navid

Kimovski, Dragi

Kinateder, Harald

Kinoshita, Tamotu

Kiverstein, Julian

Kjelstrup, Signe
Wallace, David

Wan, Feng

Wang, Anzhong

Wang, Deng

Wang, Dong

Wang, Jinfeng

Wang, Ligong

Wang, Ling

Wang, Lin-Shu

Wang, Mei

Wang, Shibing

Wang, Shuangfeng

Wang, Wenwu

Wang, Xiaofang

Wang, Yang

Wartena, Christian

Watanabe, Eiichi

Watanabe, Kazuho

Waterstraat, Nils

Way, Michael J.

Weber, Stefan

Wedemann, Roseli S.

Weijs, Steven

Weinert, Friedel

Wernik, Jacek

West, Bruce J.

Westerbäck, Thomas

Wharton, Ken

Whitaker, M.A.B. (Martin Andrew)

White, Martin

White, Roger

Wibisono, Andre

Wibowo, Santoso

Wieczorek, Piotr

Wiederholt, Mirko

Wieland, Christoph

Wilk, Grzegorz

Wilson, Christopher G.

Wilson, Eddie

Winther, Ole

Wolf, Alex

Wolf, Christian

Wolpert, David

Wong, Ka-Chun

Wong, Lawrence

Wong, M.L. Dennis

Woo, Jae Oh

Woodbury, Allan D.

Woodhead, Erik

Wootters, William

Woźniak, Marcin

Wrzesiński, Dariusz

$\mathrm{Wu}$, Hau-tieng 
Klados, Manousos

Kleidis, Kostas

Klein, Tony

Klika, Václav

Klimenko, Alexander

Klimenko, Alexander Y.

Knuplesch, David

Knuth, Kevin

Ko, Wen-Chang

Koch, Tobias

Kochanek, Krzysztof

Kochubei, Anatoly

Kohar, Vivek

Kolchinsky, Artemy

Kolkiewicz, Adam W.

Komabayashi, Tetsuya

Komiyama, Junpei

Kong, Wanzeng

Kononovicius, Aleksejus

Konstantas, Dimitri

Konstantinou, Georgios

Korbel, Jan

Kordos, Mirosław

Korzekwa, Kamil

Korzhik, Valery

Kostina, Victoria

Kostopoulos, Spiros

Kotsiantis, Sotiris

Kouchaki, Samaneh

Koutsouris, Dimitris

Kowalski, Frank V.

Kowalski, Gregory

Krajewski, Jarek

Krasnov, Oleg A.

Kravtsov, Sergey

Kremer, Gilberto

Kudryashov, Boris D.

Kuffer, Monika

Kugiumtzis, Dimitris

Kukreja, Muskan

Kulik, Rafal

Kumagai, Atsuya

Kumamoto, Kazuo

Kuperberg, Greg

Kurabayashi, Shuichi

Kurihara, Seiji

Kurniawan, Ernest

Kuś, Marek

Kuzuoka, Shigeaki

Kwak, Ho-Young

Kwiecień, Joanna

Kyprianidis, I.M.

La Manna, Marco
$\mathrm{Wu}, \mathrm{Jui}-\mathrm{yu}$

$\mathrm{Wu}$, Shuen-De

$\mathrm{Wu}$, Xiaoqun

Wu, Ximing

Xavier, Antonio

Xiao, Boqi

Xiao, Liangliang

Xiao, Ming

Xie, Hongbo

$\mathrm{Xu}$, Dianguo

Xu, Haiyan

$\mathrm{Xu}$, Mingtian

$\mathrm{Xu}$, Shu

Xu, Xiaoming

$\mathrm{Xu}$, Zelin

Xue, Xianwu

Yablonsky, Gregory

Yamada, Toru

Yamagishi, Masao

Yan, Zhiyuan

Yang, Fan

Yang, Hyun Jong

Yang, Jason

Yang, Jianhua

Yang, Min-Hsiung

Yang, Sheng

Yang, Shengtian

Yang, Shiming

Yang, Tian-Zhi

Yang, Yuan

Yang, Zhi-Xin

Yano, Keisuke

Yanushkevich, Svetlana

Yatsalo, Boris I.

Ye, Jun

Ye, Zhisheng

Yentes, Jenna M.

Yeo, Hwasoo

Yetis, Cenk M.

Yi, Sang-Hoon

Yi, Xinping

Yin, Penghang

Ying, Mingsheng

Yook, Soon-Hyung

Yoon, Joon Yong

Yoon, Peter H.

Youk, Hyun

Yu, Hak Ki

Yu, Keming

Yu, Meichen

Yu, Zeyun

Yuasa, Kazuya

Yue, Yuanzheng 
Lai, Lifeng

Laio, Alessandro

Lake, Douglas E.

Lamata, Lucas

Lambrou, Tryphon

Lampart, Marek

Land, Martin

Langer, Andreas

Langley, Philip

Laroche, Beatrice

Larrarte, Frédérique

Larson-Prior, Linda J.

Latella, Ivan

Laudisa, Federico

Laurent, Mevel

Lavasan, Arash Alimardani

Lawyer, Glenn

Lazaro, Jesus

Le Clainche, Soledad

Lê, Hông Vân

Lebrun, Jane

Ledrappier, François

Lee, Eon Soo

Lee, Gihyoun

Lee, Ming-Tsang

Lee, Suk Jin

Lee, Tae H.

Leff, Harvey S

Lefkidis, Georg

Lehman, Joel Anthony

Leike, Reimar $\mathrm{H}$.

Lelea, Dorin

Lemus, Enrique

Lenzi, Ervin Kaminski

Leo, Marco

León, Gabriel

Leone, Antonio

Leonelli, Cristina

Leonski, Wieslaw

Lerga, Jonatan

Lerma, Claudia

Leroux, Camille

Letelier, Juan Carlos

Leung, Carson

Leyvraz, François

Lezoray, Olivier

Li, Chang-Tsun

Li, Duan

Li, Guanchen

Li, Huaizhong

Li, Peter

Li, Qianqian

Li, Rongjian

LI, Ruiying
Yuen, Chau

Yuen, Ka-Veng

Yunusa-Kaltungo, Akilu

Zagorecki, Adam

Zagouras, Athanassios

Zahidi, Karim

Zahorian, Stephen A.

Zambrano, Davide

Zampieri, Marcos

Zanella, Mattia

Zangi, Ronen

Zanin, Massimiliano

Zavadskas, Edmundas Kazimieras

Zbeeb, Khaled

Zboril, Frantisek

Zdunek, Rafal

Zelevinsky, Vladimir

Zema, Demetrio Antonio

Zenil, Hector

Zhan, Haifei

Zhang, Bowu

Zhang, Haochun

Zhang, Le

Zhang, Pan

Zhang, Qi

Zhang, Tonghua

Zhang, Wei-Qiang

Zhang, Xiaoguang

Zhang, Xie

Zhang, Yangjun

Zhang, Yi

Zhang, Yu

Zhang, Yudong

Zhang, Yushu

Zhang, Zhenjiang

Zhao, Cheng

Zhao, Hangbo

Zhao, Jichao

Zhou, Bi-Cheng

Zhou, Huiyu

Zhou, Liang

Zhou, Siyi

Zhu, Lingjiong

Zhu, Mingyao

Zhu, Shunpeng

Zhu, Yong

Zhu, Yungang

Zieliński, Wojciech

Ziemke, Tom

Zimmer, Johannes

Zmeskal, Oldrich

Zonta, Francesco

Zorzi, Mattia

Zosso, Dominique 
Li, Tiancheng

Li, Wentian

Li, Xiang

Li, Xiaodong

Li, Xiaoli

Li, Yingsong
Zotin, Alexey

Zou, Yue-Xian

Zubiaga, Arkaitz

Zunino, Luciano

Zusai, Dai

Zylberberg, Joel

(C) 2018 by the author. Licensee MDPI, Basel, Switzerland. This article is an open access article distributed under the terms and conditions of the Creative Commons Attribution (CC BY) license (http://creativecommons.org/licenses/by/4.0/). 